\title{
INTRODUCTORY TALK
}

\author{
R. J. TAYLER
}

Astronomy Centre, University of Sussex, Sussex, England

In this talk I shall try to outline the subject which will be discussed in detail by the succeeding speakers. I shall concentrate on ideas and I shall not attempt to present any definite results. The two sides to this problem are:

(a) How much helium is there in the Universe? and

(b) Where and how has it been produced?

I shall sub-divide (a) and (b) into

(a) (i) Direct observations

(ii) Inferences from theories of stellar structure and evolution

(b) (i) Helium production in stars

(ii) Primeval helium production

(iii) Production in other objects

\section{(a) (i) Direct Observations of Helium Abundance}

Direct observations of helium are difficult because the low-lying lines of neutral helium are absorbed by interstellar hydrogen. Hence it is only possible to observe helium in hot objects in which higher lines of neutral helium or ionized helium are visible. In these cases there may be difficulties because of departures from LTE; although helium was first discovered in the Sun, there is no reliable direct measure of its helium abundance. Helium abundances can be obtained for:

$(\alpha)$ hot main-sequence stars,

$(\beta)$ horizontal branch stars and subdwarfs,

$(\gamma)$ gaseous nebulae,

$(\delta)$ planetary nebulae.

It is important to consider what significance these abundances have. Although it is possible to obtain a good estimate of the present helium abundance of the interstellar medium from $\mathrm{O}$ and $\mathrm{B}$ stars and from gaseous nebulae like the Orion nebula, it is not possible to obtain a direct measurement of the helium content of old unevolved stars. Some old evolved stars can be studied, such as some hot subdwarfs and horizontal branch stars as well as planetary nebulae. If such objects are observed to have high helium content, it may not mean that they had high helium content when they were formed because they may have suffered mass loss and mixing in their earlier evolution but, if they have low helium content and no other obvious abnormalities now, it is probable that they had low helium initially. Such an observation would be regarded as direct evidence that the initial helium content was low in at least part of the Galaxy. 
What are the actual results which have been obtained? Abundances in young stars and gaseous nebulae have generally been found to be in the range $25 \%$ to $40 \%$ by mass with the majority of values being in the lower part of the range and none significantly lower than $25 \%$. Such results have been obtained from optical measurements and from radio recombination lines in gaseous nebulae and the evidence is suggestive that the helium content of the interstellar gas today is generally greater than $25 \%$ by mass.

The values obtained for planetary nebulae are not significantly different. This may mean that the parent stars of the planetary nebulae were born with a substantial helium content, but it may alternatively mean that the stellar mass loss, which is the planetary nebula phenomenon, is accompanied by substantial mixing of recently synthesised helium to the surface.

There has been considerable dispute in recent years about the helium content of certain horizontal branch and similar stars. There have been several reports that some of these stars have essentially no helium, and this would have very important consequences if it were true, but most of these reports have subsequently been withdrawn by their authors. As $\mathrm{I}$ at present understand it, nobody feels absolutely certain that any of these stars is substantially helium deficient, but possibly Dr. Cayrel will be able to present up-to-date information on this point.

Subject to this important uncertainty about the halo horizontal branch stars, it is possible that all objects in our Galaxy have upwards of about $25 \%$ helium by mass, but it must be reiterated that we cannot observe the helium (if present) in old unevolved stars. A similar helium abundance has been observed in some gaseous nebulae in nearby galaxies, but there has been a report of a very much lower helium content in a quasar and this will be mentioned again later.

As mentioned earlier, there is no direct measurement of the helium content of the Sun but an indirect value can be obtained from a study of solar cosmic rays. The $\mathrm{He}: \mathrm{C}: \mathrm{N}: \mathrm{O}$ ratios in the cosmic rays can be compared with the $\mathrm{H}: \mathrm{C}: \mathrm{N}: \mathrm{O}$ ratios in the photosphere. Since $\mathrm{He}, \mathrm{C}, \mathrm{N}, \mathrm{O}$ nuclei have the same charge to mass ratio they are probably accelerated similarly in the cosmic rays and a comparison of the above ratios should enable a solar helium abundance relative to hydrogen to be deduced. There has in recent years been a downward revision of the solar CNO abundances and current $\mathrm{He}$ abundance deduced in this manner is rather less than $25 \%$. Is this value significant?

\section{(a) (ii) Inferences from Theories of Stellar Structure and Evolution}

We now turn to indirect estimates of helium abundances obtained from theories of stellar structure and evolution. There are a variety of such determinations and Dr. Faulkner will be discussing the subject in detail. Some of them are as follows:

( $\alpha$ ) PROPERTIES OF VARIABLE STARS

Non-linear calculations of the structure of variable stars have in the past few years 
gone a long way towards describing not only the gross properties of the stellar pulsation, but also details such as shapes of light curves including the positions of secondary humps on the light curves. Studies of both RR Lyrae stars and cepheids have failed to obtain results which are in good agreement with observations unless a rather substantial helium content is assumed $(30 \%)$. Cepheids are young stars and the value found for them is not greatly in excess of that observed in other young objects. The RR Lyrae value is probably more significant although, as with the other horizontal branch stars mentioned earlier, it need not necessarily reflect the original composition of these old stars.

\section{( $\beta$ ) THE STRUCTURE OF THE SUN}

For many years past, attempts have been made to produce models of the Sun which possess all of its observed properties. Free parameters in these models are the chemical composition and age of the Sun. Of course neither of these is completely free. The age of the Sun is probably rather accurately known and the biggest uncertainty is probably the solar helium content (there are of course uncertainties of other types, including inaccuracies in the laws of opacity and energy transport by convection). A few years ago it was believed that the present properties of the Sun could only be explained by a fairly high helium content $(\approx 28 \%)$. More recently there has been doubt about this result. The failure of the solar neutrino experiment to give results which agreed with theoretical predictions has meant that there must be something wrong with previously obtained solar models. The attempt to produce new solar models, which are consistent with the observed neutrino flux and which agree with the other solar properties, has cast some doubt on the high helium content as well as causing fundamental questions to be asked about the theory of stellar structure. At present I believe that there is no clear agreement between all workers in the field about the required helium abundance.

\section{( $\gamma)$ SHAPE OF HR DIAGRAMS OF GLOBULAR CLUSTERS AND OLD GALACTIC CLUSTERS}

This is perhaps potentially the most important source of information of this type as the globular cluster stars are old stars. If the helium content can, for example, be determined by study of the structure of the HR diagram below the top of the giant branch where the helium flash occurs, this should certainly give information about the original chemical composition of the globular cluster stars. Most attempts to account for the shape of the sub-giant branch of the globular cluster HR diagram and for properties of stars on the horizontal branch have suggested that globular cluster stars should have a relatively high helium content $(\sim 30 \%)$ but even here there are some dissenting voices. Dr Faulkner has made a special study of this problem and I imagine that he can give us some up-to-date estimate of the helium content of globular cluster stars.

It should be clear from what I have said that no one of these deductions from the theory of stellar structure is completely secure but, uncertain as the individual determinations are, they mainly lead to a rather high helium content. 


\section{(b) (i) Helium Production in Stars}

We now turn to the production of helium and ask whether all of the helium in the Galaxy could have been produced in stars in the lifetime of the Galaxy. Here it must be understood that we are interested in the useful production of helium which gradually enriches the interstellar medium and which causes a change in the chemical composition of stars at birth rather than the total production of helium which includes all of the helium retained inside the stars in which it is produced. There are considerable uncertainties in this subject, a principal one being that mass loss from stars is not at all well understood at present. Clearly it must be difficult to answer the question unambiguously while we do not know whether all stars in the Galaxy have a high helium content or whether only the young stars have a high helium content, while the oldest stars have very little helium.

However, the following general points can be made:

$(\alpha)$ If the galactic luminosity has not changed considerably during its lifetime, the total helium production is probably less than $5 \%$ of the galactic mass. This value is obtained by assuming that essentially all of the galactic luminosity is produced by the conversion of hydrogen to helium. Much of this helium would be locked up inside stars and, even if only relatively young objects have substantial amounts of helium, it does not seem possible that anything like the complete amount could be produced in this way.

$(\beta)$ The Galaxy might have been much more luminous in the past because of early generations of massive stars with high luminosity to mass ratio. At first sight it appears that a large amount of helium can be produced very rapidly in such stars, but there is a problem. In massive stars the conversion of hydrogen to helium is followed by other reactions converting helium into heavier elements. At any time the total amount of unburnt helium is limited and, if an instability occurs liberating the helium into the interstellar medium, it is difficult to see why a comparable amount of heavier elements is not liberated. If such massive stars were the main producers of helium, why is there of the order of ten times as much helium as heavy elements?

$(\gamma)$ Provided that the helium content of the Galaxy has increased gradually from an initial low value to the present concentration in the interstellar medium (which would be supported if the solar helium content turned out to be well below $20 \%$ by mass) it is just possible that most of the helium could have been produced in stars of 2 to $3 M_{\odot}$ which can evolve well within the galactic lifetime and probably become unstable before substantial conversion of helium to heavier elements occurs, but this would require preferential production of stars in this mass range early in the galactic lifetime.

On balance, I feel that it is difficult to believe that all of the helium in the Galaxy has been produced in stars in the galactic lifetime, but I shall look forward to hearing Dr Kippenhahn's views on this point later today. 


\section{(b) (ii) Primaeval Helium Production}

The feeling that stars could only have changed the overall helium content of the Galaxy by a few per cent and the lack of definite knowledge that any objects in the Galaxy have a helium content less than about $25 \%$, led various authors to suggest that the original chemical composition of the Galaxy must have been about $75 \% \mathrm{H}$ and $25 \% \mathrm{He}$. This suggestion was strengthened by the discovery in 1965 of the background microwave radiation and the realisation that both the microwave background and the correct amount of helium were predicted by the simplest version of the hot Big Bang cosmological theory, which assumes that the Universe is, and has been, both homogeneous and isotropic on a large enough scale. It immediately became attractive to suppose that about $25 \%$ of helium by mass was produced cosmologically and that younger objects in the Galaxy have a higher helium content because of the gradual enrichment of the interstellar medium due to helium production in stars in the galactic lifetime. This enrichment is not much greater than the enrichment in heavy elements.

In the past few years, consideration of this problem has concentrated on the following questions:

$(\alpha)$ Are there any normal stars with a very low helium abundance? As I said when I was discussing the observations, definite identification of such stars would be very important and would render a theory of cosmological helium production invalid.

( $\beta$ ) Are there significant departures from black body form in the background radiation? According to theoretical calculations, if it is relict radiation from an initial Big Bang, it should show only relatively small departures from black body form. There have been some observations which suggest that it departs substantially from black body form in the far infra-red, but my present understanding (which may be contradicted today) is that the results are still not definitely established.

In addition, the discovery of many galaxies with strong infra-red emission and, by extrapolation, strong microwave emission has led to attempts to discover whether a superposition of discrete extragalactic sources could produce

(1) The spectrum

(2) The intensity

and (3) The isotropy

of the observed radiation. It seems difficult to produce (2) and (3) without an implausible number of sources. Personally I have always felt that the strongest argument for the cosmological origin of the radiation (and against any superposition, galactic or extra-galactic) is that black body radiation must have both the correct spectrum and the correct energy density.

$(\gamma)$ Are there any other observations which make the cosmological theory invalid? Perhaps the most difficult problem has been to account for galaxy formation in a universe which is initially strictly homogeneous.

Clearly it is necessary to consider the helium production and the background radiation in a variety of cosmological theories, while there remains the possibility 
that the initial helium content was low and that the microwave radiation might be due to a superposition of discrete sources. Helium production in cosmological theories will be discussed today by Dr Wagoner and Dr Novikov. At present it suffices to say that there are cosmological theories which produce:

(1) Helium, but no microwave background:

Some versions of the Cold Big Bang.

(2) Microwave background, but little helium:

Some anisotropic Hot Big Bang theories and Hot Big Bang theories with neutrino degeneracy.

(3) No helium or microwave background:

Steady State and other theories involving continuous creation.

Of course, as mentioned earlier, these two aspects of cosmological theories cannot be considered in isolation from all others.

\section{(b) (iii) Helium Production in Objects other than Stars}

Finally we consider the possibility that helium has been produced in the Galaxy during the galactic lifetime but not in ordinary stars. Most of the objects for which we have reliable abundances are Population I objects in, or near to, the plane of the Galaxy. Most of the objects for which a low helium abundance has been conjectured are halo objects. Perhaps the old halo objects do have a low helium abundance while old objects from near the galactic centre may not have a low helium abundance. Is it conceivable that there are important spatial variations in the chemical composition of the Galaxy? Recent results have suggested that this may be true as far as the metals are concerned. This leads to the idea that massive objects in the centre of the Galaxy (little bangs of a type I expect Dr Wagoner to describe) may have been responsible for significant nucleosynthesis. This would accord with the increasing evidence for explosive events in galactic nuclei and it might tie in with the observation of a low helium abundance in the outer region of a quasar.

\section{Concluding Remarks}

As 1 said at the beginning of this talk, my object this morning has been to set the scene for this discussion and to open as many doors as possible. It may be that some of them will be closed by the subsequent speakers and we shall look forward to hearing from Professor Burbidge at the end of the meeting whether he thinks that any definite results have emerged. 